\title{
Statistical Image Reconstruction Methods in PET with Compensation for Missing Data
}

\author{
PE Kinahan ${ }^{1}$, JA Fessler ${ }^{2}$, and JS Karp 3 \\ Universities of ${ }^{1}$ Pittsburgh, ${ }^{2}$ Michigan, and ${ }^{3}$ Pennsylvania
}

\section{Abstract}

We present the results of combining volume imaging with the PENN-PET scanner with statistical image reconstruction methods such as the penalized weighted least squares (PWLS) method. The goal of this particular combination is to improve both classification and estimation tasks in PET imaging protocols where image quality is dominated by spatiallyvariant system responses and/or measurement statistics. The PENN-PET scanner has strongly spatially-varying system behavior due to its volume imaging design and the presence of detector gaps. Statistical methods are easily adapted to this scanner geometry, including the detector gaps, and have also been shown to have improved bias/variance trade-offs compared to the standard filtered-backprojection (FBP) reconstruction method. The PWLS method requires fewer iterations and may be more tolerant of errors in the system model than other statistical methods. We present results demonstrating the improvement in image quality for PWLS image reconstructions of data from the PENN-PET scanner.

\section{INTRODUCTION}

\section{Statistical Image Reconstruction Methods.}

It is well recognized that statistical reconstruction algorithms, such as ML-EM, are particularly helpful in cases where measurement statistics dominate image quality [1]. To realize their full potential, these algorithms depend on accurate system models that describe the system response function and expected measurement statistics for a known object.

Statistical iterative methods assume that the expected value of the projection data is given by $\overline{\mathbf{y}}=\mathrm{E}[\mathbf{y}]=\mathbf{A x}$, where $\mathbf{x}=\left\{x_{i} \mid i=1, \ldots, n\right\}$ is a vector containing the $n$ voxel values of the true image, $\mathbf{y}=\left\{y_{j} \mid j=1, \ldots, m\right\}$ contains the $m$ measured projection values, and $\mathbf{A}=\left\{A_{i j}\right\}$ is the $m \times n$ system matrix that gives the probability of a photon emitted from voxel $i$ being detected in projection bin $j$. The measured projection data, on the other hand, is a random vector drawn from the probability distribution function (PDF) given by an assumed statistical model. The ML-EM method assumes that the system model, A, accurately relates the PDF of the estimated image data to the Poisson-distributed sinogram data and seeks to maximize the log likelihood, possibly with the inclusion of an a priori regularizing term included via a maximum a posteriori (MAP) formulation of the estimation problem.

The expected measurement statistics are usually assumed to be Poisson, often with the inclusion of attenuation and other

\footnotetext{
${ }^{1}$ Department of Radiology, 200 Lothrop Street, Pittsburgh, PA, 15213. This work was supported by NIH grant CA 60711 and DOE grant DE-FG02-88ER60642.
}

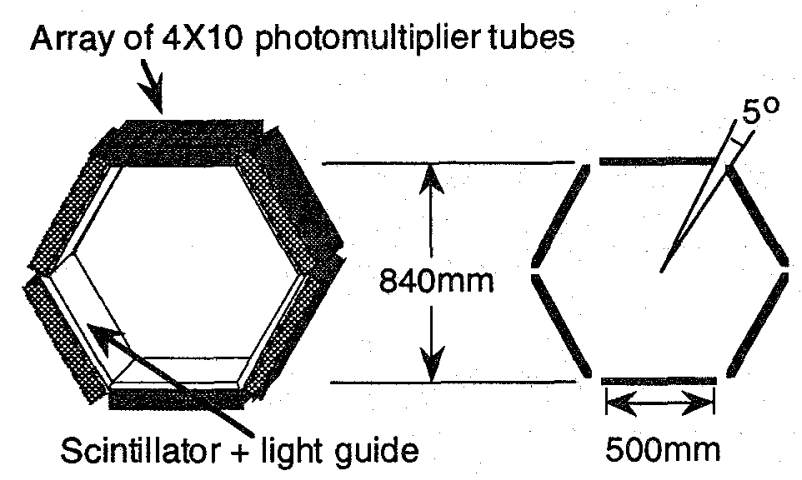

Fig. 1. Geometry of the PENN-PET scanner.

effects, and the system response function is usually assumed to spatially invariant. Neither of these assumptions are completely accurate in practice. An alternative approach is to assume Gaussian statistics and minimize a penalized weighted least squares (PWLS) objective function, rather than a Poisson likelihood objective function [2]. The PWLS objective function, essentially a regularized $\chi^{2}$ functional, is given by,

$$
\Phi(\mathbf{x})=-\sum_{j}\left(\sum_{i} A_{i j} x_{i}-y_{j}\right)^{2} \sigma_{j}^{-2}-\beta U(\mathbf{x}),
$$

where $\sigma=\left\{\sigma_{j} \mid j=1, \ldots, m\right\}$ are the standard deviations for the projection data and $\beta$ controls the influence of the regularizing prior, $U(\mathbf{x})$, which is described elsewhere [6]. The standard deviations are estimated based on the corrections applied to the raw sinogram data during the processing steps prior to the image reconstruction, and the effect of weighting the differences between estimated and measured projections takes into account the statistical quality of the data. The effect of the correction steps on the weights are described below.

Minimization of a PWLS objective function by successive over-relaxation (SOR) has been shown to be effective in cases where the data statistics are not Poisson [2]. The SOR algorithm is a coordinate-descent method where image pixels are estimated in place. In other words, $\Phi\left(x_{i}\right)$ is minimized independently for each image pixel, $i$, in turn. This has the advantages of estimating the image data directly, and allowing for the straightforward application of a non-negativity constraint on the estimated image.

\section{The PENN-PET Scanner System Model}

The PENN-PET scanner at the University of Pennsylvania is a volume-imaging PET camera with an axial field of view (FOV) of $12.8 \mathrm{~cm}$, a transverse FOV of $50 \mathrm{~cm}$ and gaps in coverage of $\sim 5 \mathrm{deg}$ where the 6 planar detectors meet as illustrated in fig. 1 [3].

Since the scanner has no septa, data are acquired in 3D mode, with on-line rebinning of the 3D data into standard $2 \mathrm{D}$ sinograms via the single-slice rebinning (SSRB) algorithm 
[4]. The gaps in the detector coverage lead to the missing sinogram regions shown in fig. 2 .

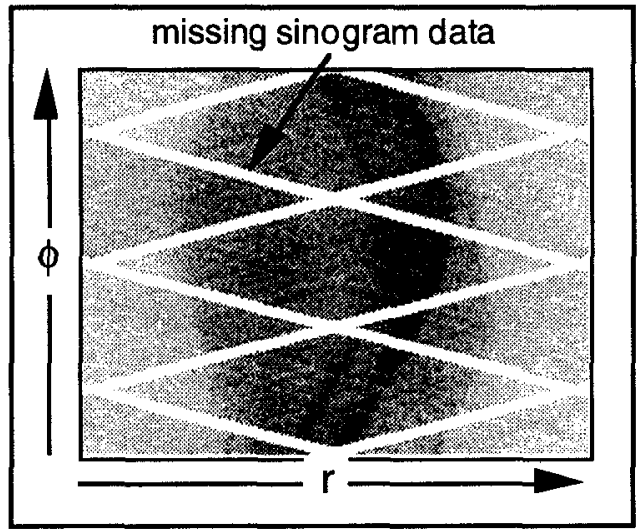

Fig. 2 Missing sinogram data due to $5^{0}$ detector gaps.

The corrected sinogram data, $\mathbf{y}_{c}$, can be described by:

$$
\mathbf{y}_{c}=\mathbf{a} \cdot\left(\mathbf{e}_{1} \cdot \mathbf{e}_{2} \cdot \mathbf{e}_{3} \cdot \mathbf{y}_{S S R B}-\mathbf{b}\right)
$$

where $y_{S S R B}$ is the SSRB rebinned sinogram data, $b$ is the fitted scatter and randoms subtraction [3], $\mathbf{a}$ is the attenuation correction, and $e_{i}$ are efficiency corrections for $i=1$ : axial variation due to $S S R B$ rebinning of volume acquisition, $i=2$ : sampling pattern from on-line rebinning, and $i=3$ : detector efficiency variations.

For conventional filtered-backprojection (FBP) image reconstruction, the constrained Fourier space method (CFSM), based on consistency conditions in the Fourier transform of the sinogram data, is used to estimate the missing sinogram data $[3,5]$.

Statistical reconstruction methods are easily adapted to missing data situations, especially if there are still more data samples than image pixels. These methods can directly incorporate constraints and prior information to partially compensate for the missing rays, unlike FBP, which must start with a complete data sinogram. The PWLS+SOR method is particularly well suited to reconstruct data from the PENN-PET scanner, as the weights associated with the regions of missing sinogram data can simply be set to zero. In addition, the PWLS+SOR may be more tolerant than other statistical methods of inaccuracies in the system model, such as those introduced by the SSRB rebinning and subsequent renormalization steps, since it only takes into account the mean and variance of the sinogram data, and does not expect the entire probability distribution function (e.g. a Poisson distribution) to be characterized.

\section{ESTIMATION OF MISSING DATA}

To compare the effectiveness of the PWLS+SOR and CFSM+FBP methods in reconstructing the incomplete data from the PENN-PET scanner, images were reconstructed of a simulated cylinder. The simulation did not include any other effects than Poisson noise and detector gaps. The reduction in statistical noise is shown in figs. 3 and 4 . The missing sinogram data are effectively compensated for with both methods, as there is sufficient frequency information to for the CFSM algorithm to estimate the missing sinogram data [5].

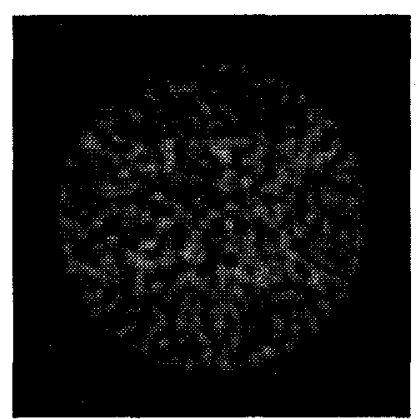

Fig. 3a. Simulated uniform cylinder data reconstructed by CFSM+FBP method.

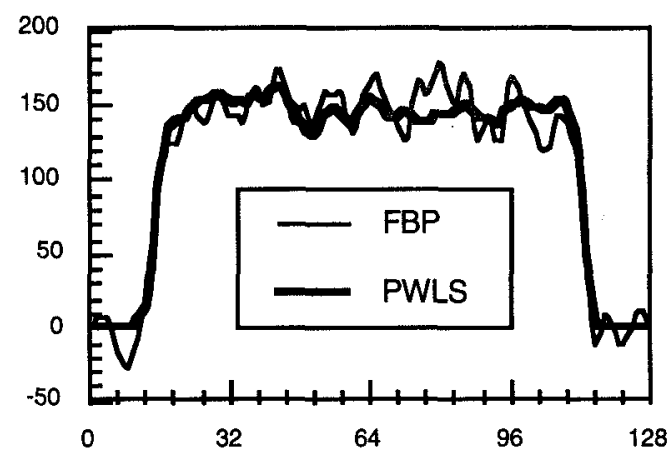

Fig. 4. Transverse line profiles through the center of the images (shown above) reconstructed by FBP and PWLS. Pixels are $2 \mathrm{~mm}$.

Figures 3 and 4 also illustrate a reduction in the image noise for the PWLS+SOR method for similar image resolutions as the CFSM+FBP image, as shown by the profiles at the edges of the cylinder.

\section{POINT SPREAD FUNCTION}

Imaging a point source is more challenging than a cylinder as there are more missing spatial frequencies in the sinogram data. To investigate the effects on the PSF, a slightly offcenter line was imaged in air and reconstructed without applying any corrections for scatter or attenuation. The reconstructed images were carefully matched between the two methods to have the same FWHM $(11.8 \mathrm{~mm})$ and FWTM $(24.6 \mathrm{~mm})$, which are typical of clinical whole-body imaging, and is the same resolution used in the phantom and patient images below. Surface plots are shown in fig. 5 along with plots are zeroed out to the FWTM level to show detail in the tails.

The increased fluctuations in the tails for the CFSM+FBP method are evident in fig. 5. To quantify the magnitude of the fluctuations in the tails of the PSF, Fig. 6 shows the integrated counts for an ROI of a given diameter as a fraction of the total counts in the image.

In the original image, the fraction approaches 1 more quickly for the CFSM+FBP method. This, however is due to the offsetting negative swings in the tails, and the PWLS+SOR PSF actually has tails of a smaller magnitude, as shown by applying the same measure to the image with the negative values truncated to zero. 

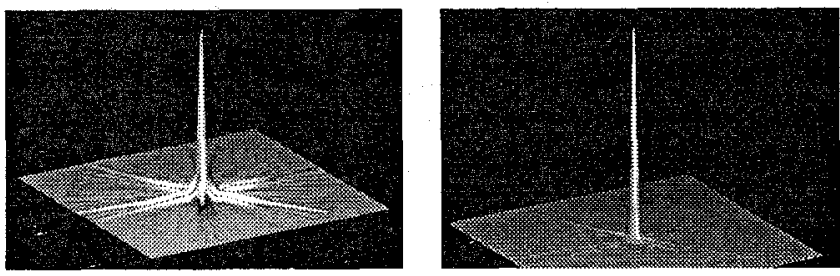

Line source reconstructed by $\mathrm{CFSM}+\mathrm{FBP}$

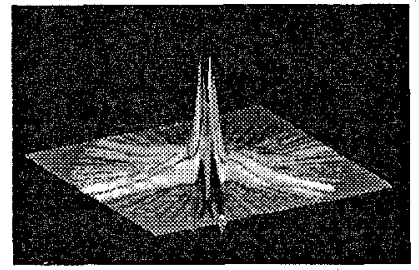

CFSM+FBP image zeroed to the FWTM

Line source reconstructed by PWLS+SOR

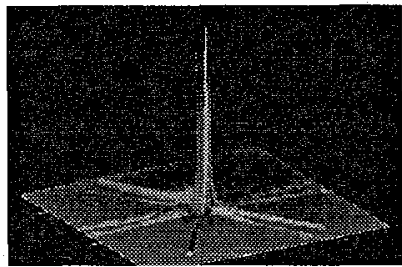

PWLS+SOR image zeroed to the FWTM

Fig 5. Map of central image plane of a line source reconstructed by both methods with equal resolution as determined by FWHM and FWTM. The bottom pair show the same images set to zero out to the FWTM level in order to show detail in the tails of the PSF.

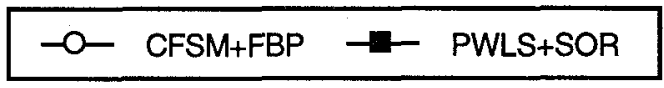

Fraction of total image value vs. ROI radius

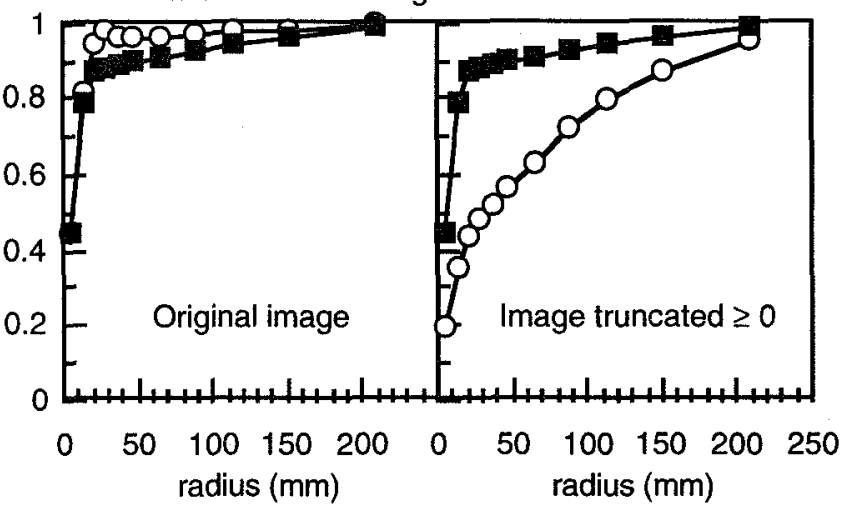

Fig 6. Measure of the magnitude of the tails of the PSF.

\section{RESOLUTION, CONTRAST, AND NOISE}

To compare both the effects of the reduced tails in the PSF with the expected noise reduction properties of the PWLS+SOR method, a cylinder with a hot line source (offcenter), a warm background and a cold cylindrical insert was imaged on the PENN-PET scanner to simultaneously measure resolution, contrast, and noise. The phantom was scanned for two different imaging conditions, one of low statistics $(\sim 50 \mathrm{k}$ events per image slice), and one or moderate statistics $(\sim 250 \mathrm{k}$ events per image slice). The images were reconstructed over a range of parameters to control the usual bias/resolution tradeoff. For the CFSM+FBP method this was done by setting the cut-off frequency of the apodizing Hamming window to 2.0, $1.0,0.67,0.5$, and 0.4 of the Nyquist frequency $\left(0.25 \mathrm{~mm}^{-1}\right)$, while for the PWLS+SOR method this was done by controlling the influence of the regularizing term by setting the parameter $\beta$ in equation (1) to $2^{-\mathrm{q}}$, where $\mathrm{q}=14.0,12.8$, $9.0,7.0,5.9$, and 4.7. For the SOR minimization algorithm,

20 iterations were used, although 10 iterations would likely have given similar results.

In addition, images reconstructed using the ordered-subsets ML-EM (OSEM) algorithm [7] were included for comparison. For the OSEM images the system model $\mathbf{A}$, did not take into account any non-ideal effects, such as attenuation correction. To control the bias/resolution trade-off, 8 subsets were used and the number of iterations was varied from 1 to 4 , followed by post-reconstruction smoothing with a $3 \times 3$ Gaussian filter with a $8 \mathrm{~mm}$ FWHM.

The PSF of a PET scanner (with or without detector gaps) is not well characterized by estimating a FWHM from a fitted Gaussian profile. This is especially true for asymmetric PSFs of off-center points. For our purposes resolution was calculated using,

$$
F W H M=\sqrt{\left(F_{W H M}^{2}+F W H M_{T}^{2}\right) / 2},
$$

where FWHM $_{R}$ and $\mathrm{FWHM}_{\mathrm{T}}$ are the radial and tangential FWHM directly measured using linear interpolation on profiles through the reconstructed line source. A similar measure was used to determine the FWTM.

Contrast was measured from ROIs of the same diameter as the cold cylindrical insert placed over the background and the cold insert (determined from a high statistics scan), and calculated as the ratio (background - cold)/background.

Noise was calculated as the standard deviation of the background or cold ROIs normalized to the background level. The validity of this type of measurement is discussed below.

\section{Resolution versus Noise}

Fig. 7 shows no significant difference between the CFSM+FBP and PWLS+SOR methods. This result is discussed in more detail below.

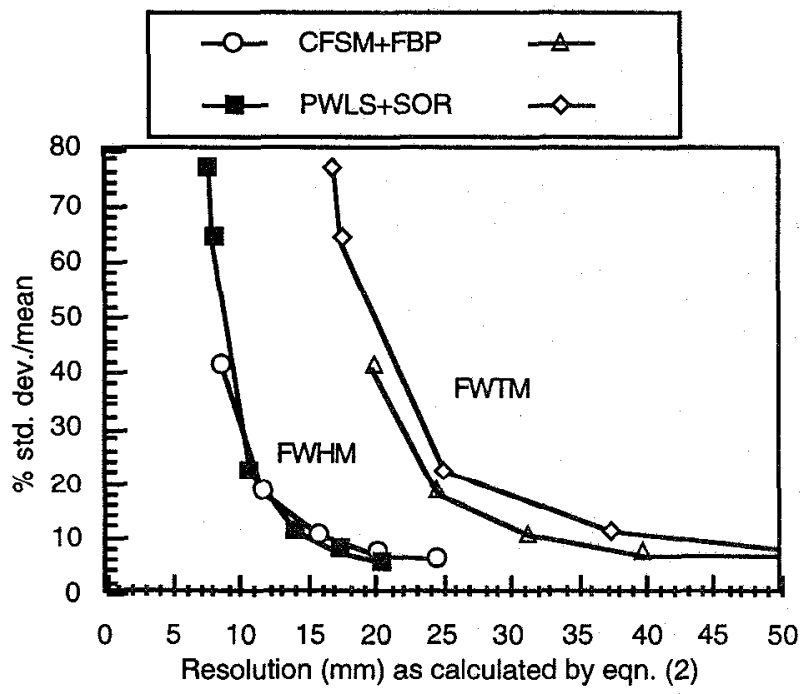

Fig. 7. Background noise vs. resolution as measured by the FWHM and FWTM of a line source in a warm cylinder.

\section{Contrast versus Noise}

Fig. 8 shows the contrast vs. noise for the background and cold regions for the low and moderate statistics cases. The OSEM method shows the expected improvement for the 
contrast/noise ratio as compared to the CFSM+FBP method in the cold ROI regions. In comparison, the PWLS+SOR demonstrates an improved contrast/noise ratio in all cases, likely due to the combination of incorporation of the statistics in the data and the reduction in the tails of the PSF demonstrated above.
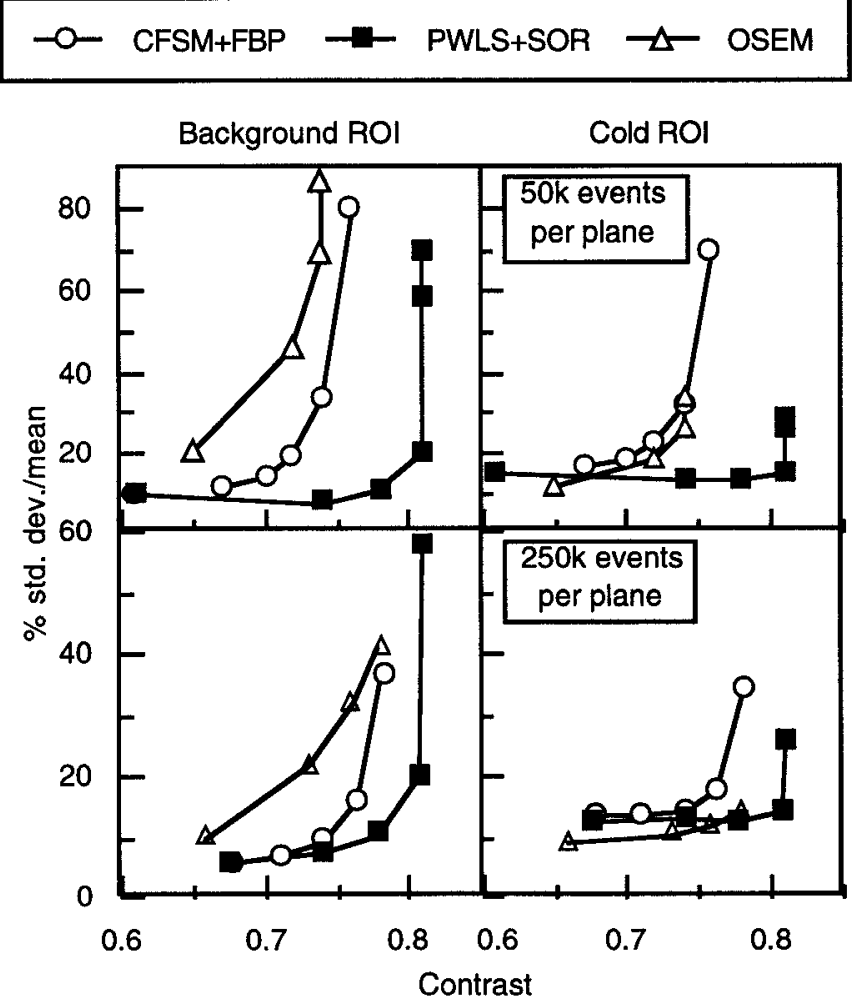

Fig. 8. Contrast vs. noise for two different imaging conditions. OSEM (without proper system model) is also included.

\section{Contrast versus Resolution}

To directly compare the effects the extended tail of the PSFs on image quality, the contrast vs. resolution for the two methods was compared. Fig. 9 explains the apparent difference in comparing resolution vs. noise (fig. 7) and contrast vs. noise (fig. 8), by showing an improved contrast of the PWLS+SOR method over wide range of resolutions.

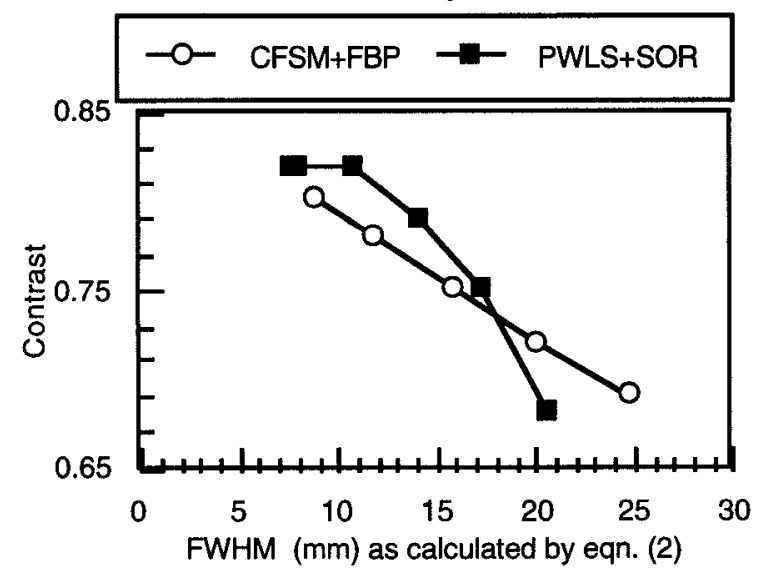

Fig. 9. Contrast vs. resolution as measured from a cylinder with a hot line source, a warm background and a cold insert.

\section{APPLICATION TO WHOLE BODY IMAGING}

\section{Phantom Study}

An anthropomorphic torso phantom with hot (10:1 and 3:1 ratios of activity to background concentrations, $1 \mathrm{cc}$ each) and a cold sphere insert $(16 \mathrm{cc})$ was imaged to examine the reduction in noise for a fixed resolution identical to that used in the PSF study above. Attenuation correction was performed using a singles transmission source [8].
CFSM+FBP

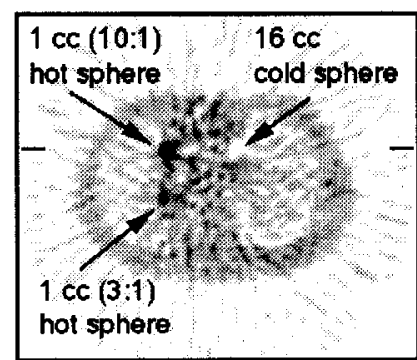

Transverse

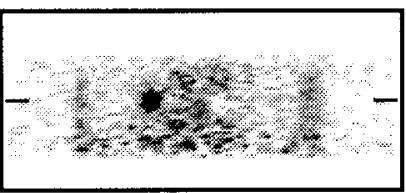

Frontal
PWLS+SOR

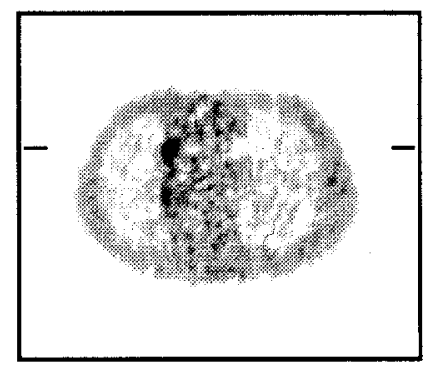

Transverse

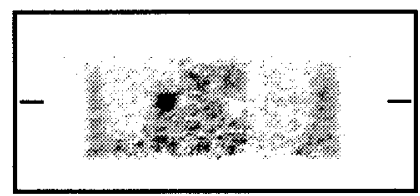

Frontal
Fig. 10. Images from a anthropomorphic phantom which has hot and cold sphere inserts. Imaging and reconstruction parameters are similar to those of the whole-body images in figs. 11 and 12 .

Figure 10 shows transverse and frontal section through images reconstructed using both methods. Images were reconstructed with identical resolution. Imaging and reconstruction parameters are similar to those of the wholebody patient study described next to give a basis for comparing the patient images. The reconstruction parameters are typical for whole-body oncology imaging, with the exception of using no axial smoothing to allow for a more direct comparison of the two algorithms.

Measured contrast and standard deviation values are given in table 1. Contrast was measured from ROIs of the same diameter as the spherical inserts placed over inserts (determined from a high statistics scan), and calculated as the ratio lbackground - coldVbackground. Pixel standard deviation was averaged from large ROIs placed in each of the 'lung' regions of the phantom.

Table 1. Contrast of spheres and standard deviation of lung regions expressed as a percentage of the background.

\begin{tabular}{lccccc}
\hline \hline \multicolumn{1}{c}{ sphere } & \multicolumn{3}{c}{ Contrast } & Std. dev. \\
& $\begin{array}{c}1 \mathrm{cc} \\
\text { hot } \\
(10: 1)\end{array}$ & $\begin{array}{c}1 \mathrm{cc} \\
\text { hot } \\
(3: 1)\end{array}$ & $\begin{array}{c}16 \mathrm{cc} \\
\text { cold }\end{array}$ & \\
& 9 & 2 & 1.0 & - \\
\hline Ideal contrast & 9.4 & 1.6 & 0.76 & $\sim 31 \%$ \\
CFSM+FBP & 6.4 & 1.8 & 0.80 & $\sim 20 \%$ \\
PWLS+SOR & 7.2 & 1.80 \\
\hline \hline
\end{tabular}


Table 1 shows higher contrast ratios for the hot and cold spheres and a lower pixel standard deviation in the lung regions of the phantom.

\section{Patient Study}

A whole body study for breast cancer recurrence with typical imaging (approximately $200 \mathrm{k}$ counts per plane) and reconstruction parameters. The CFSM+FBP and PWLS+SOR images have matched image resolution as described above.

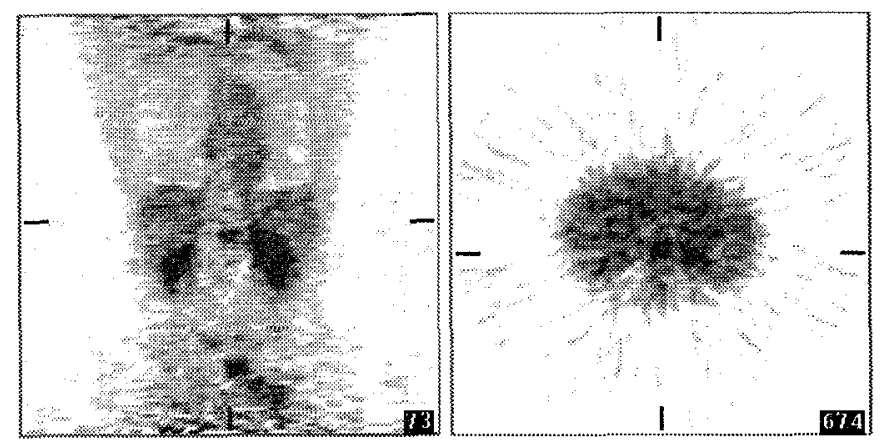

Fig. 11. Frontal and transverse views of a CFSM+FBP reconstruction of a whole body oncology study. Cross hairs are centered on a metastasis from a primary breast tumor.

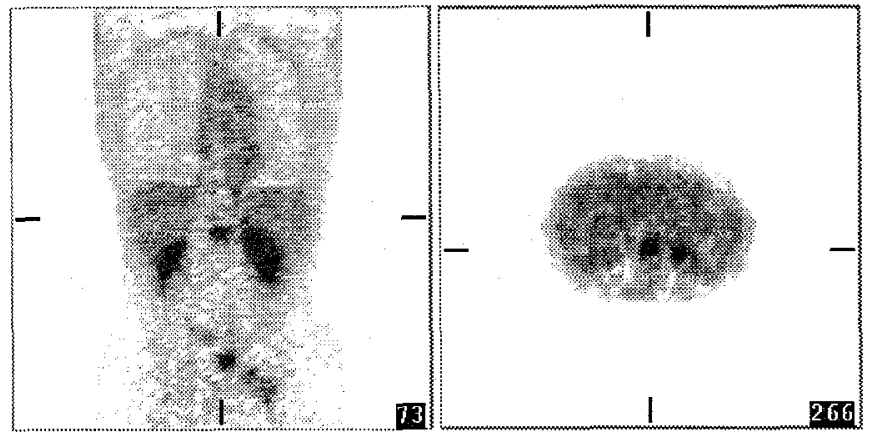

Fig. 12. PWLS+SOR images of the same whole body oncology study shown in fig. 11.

While it is not possible to make any conclusions about improved tumor detectability, figs. 11 and 12 show a reduction in statistical noise for PWLS+SOR compared to CFSM+FBP at the resolution shown above (FWHM=11.8 $\mathrm{mm}$ ), and possibly an improved contrast .

\section{DISCUSSION}

The relative reconstruction times of the algorithms are given in table 2 and show that the ratio in reconstruction times between CFSM+FBP and PWLS+SOR is only $~ 1: 3$. For the 128-plane whole-body image in fig. the reconstruction time (for 10 iterations of SOR) is only $10 \mathrm{~min}$. on an Alpha $6005 / 333$ workstation.

Table 2. Relative reconstruction times

\begin{tabular}{ll}
\hline \hline Algorithm & Relative reconstruction time \\
\hline FBP & 1 (by definition) \\
CFSM+FBP & 2.0 \\
PWLS+SOR & 6.5 (for 10 iterations of SOR) \\
OSEM & $4-8$ (dep. on num. of iterations) \\
\hline \hline
\end{tabular}

Images reconstructed with PWLS+SOR have favorable noise/contrast tradeoffs compared to those reconstructed with CFSM+FBP due to (i) reduction of the tails in the PSF and (ii) reduced propagation of statistical noise. At this point it is not clear what the relative gain from each factor is.

The tails in the PSF are reduced by PWLS+SOR likely because of positivity constraint and applying a priori smoothness constraints in image space. A caveat is that the PSF of PWLS+SOR method may have a different shape in a warm background as the positivity constraint will have less effect. The smoothness constraint, however, will still be applied in image space. The OSEM algorithm compared favorably the to CFSM+FBP method in cold regions, but less well in warm regions. It should be recalled, however, that an accurate system matrix was not used in this implementation of OSEM.

While noise calculated as the standard deviation of the background or cold ROIs does not represent the true pixel variance, it can to some extent be considered indicative of trends in image noise. The results obtained here are consistent to a simulation study (for a scanner without detector gaps) where true pixel variance was calculated from multiple realizations and showed that PWLS+SOR had improved biasnoise trade-offs in comparison to FBP [2].

The incorporation of statistical information into the PWLS+SOR method could be done by other statistical methods, such as OSEM. Given the imperfect corrections that always occur in practice, hoewever, a statistical model that is insensitive to departures from the assumed shape of the data distribution is probably preferable.

\section{ACKNOWLEDGMENTS}

We thank Gene Gualtieri from UGM Medical Systems for his assistance with the PENN-PET reconstruction software, and Robin Smith, Sean Riggin, and Emannuel Angel from the University of Pennsylvania for their assistance with data acquisition and analysis.

\section{REFERENCES}

[1] Shepp LA and Vardi Y. IEEE Trans. Med. Img. 2:113119,1982

[2] Fessler JA. IEEE Trans. Med. Img. 13:290-300, 1994

[3] Karp JS, Muehllehner G, Mankoff DA et al. J Nucl Med 31:617-627, 1990

[4] Daube-Witherspoon ME, Muehllehner G. J Nucl Med 28:1717-1724, 1987

[5] Karp JS, Muehllehner G, Lewitt RM. IEEE Trans. Med. Img. 7:21-25, 1988

[6] Fessler JA and Rogers WL. IEEE Trans. Img. Proc. 5:1346-1358

[7] Hudson HM and Larkin RS. IEEE Trans. Img. Proc. 13:601-609, 1994

[8] Karp JS, Muehllehner G, Qu H, Yan XH. Phys. Med. Biol. 40:924-944, 1995 